Pacific Journal of Mathematic 


\title{
ON A SET OF POLYNOMIALS SUGGESTED BY LAGUERRE POLYNOMIALS
}

\author{
Tilak RaJ PrabhakaR
}

Generating functions, integrals and recurrence relations are obtained for the polynomials $Z_{n}^{\alpha}(x ; k)$ in $x^{k}$ which form one set of the biorthogonal pair with respect to the weight function $e^{-x} x^{\alpha}$ over the interval $(0, \infty)$, the other set being that of polynomials in $x$.

A singular integral equation with $Z_{n}^{\alpha}(x ; k)$ in the kernel is solved in terms of a generalized Mittag-Leffler's function and a unified formula for fractional integration and differentiation of the polynomials is derived.

It is known [7] that the polynomials $Z_{n}^{\alpha}(x ; k)$ of degree $n$ in $x^{k}$ for positive integers $k$ and $\operatorname{Re} \alpha>-1$ are characterized up to a multiplicative constant by the above requirements. Konhauser [8] discussed the biorthogonality of the pair $\left\{Z_{n}^{\alpha}(x ; k)\right\},\left\{Y_{n}^{\alpha}(x ; k)\right\}$ in the basic polynomials $x^{k}$ and $x$, over the interval $(0, \infty)$ and with the admissible weight function $e^{-x} x^{\alpha}$ of the generalized Laguerre polynomial set $\left\{L_{n}^{\alpha}(x)\right\}$. Indeed the polynomials have several properties of interest and Konhauser [8] obtained among other things some recurrence relations and a differential equation for the polynomials $Z_{n}^{\alpha}(x ; k)$ which are our primary concern in this paper. For $k=2$, Preiser [11] obtained for these polynomials a generating function, a differential equation, integral representations and recurrence relations. Earlier Spencer and Fano [13] also used these polynomials for $k=2$.

For $k=1$, all the results proved in this paper reduce to those for $L_{n}^{\alpha}(x)$; in particular the integral equation (3.1) either reduces to or contains as still more special cases the integral equations solved by Widder [14], Buschman [1], Khandekar [6], Rusia [12] and Prabhakar $([10],(7 \cdot 1))$. For $k=2$, the results are essentially the same as those in [11] or [13].

2. Some properties of $\mathbf{Z}_{n}^{\alpha}(\mathbf{x} ; \mathbf{k})$. We now obtain a generating function, a contour integral representation and a fractional integration formula for $Z_{n}^{\alpha}(x ; k)$. In $\S 3$, we need the Laplace transform and in $\S 4$ derive a more general class of generating functions for the polynomials. Recurrence relations and a few other results will follow as natural consequences. We shall freely use the closed form ([8], (5))

$$
Z_{n}^{\alpha}(x ; k)=\frac{\Gamma(k n+\alpha+1)}{n !} \sum_{j=0}^{\infty}(-1)^{j}\left(\begin{array}{l}
n \\
j
\end{array}\right) \frac{x^{k j}}{\Gamma(k j+\alpha+1)}
$$


for $\operatorname{Re} \alpha>-1$; naturally the results may be established from alternative characterizations of $Z_{n}^{\alpha}(x ; k)$ but such a discussion does not seem to be of sufficient interest.

(i) A generating function. We obtain the generating function indicated in

$$
e^{t} \dot{\varphi}\left(k, \alpha+1 ;-x^{k} t\right)=\sum_{n=0}^{\infty} \frac{Z_{n}^{\alpha}(x ; k) t^{n}}{\Gamma(k n+\alpha+1)}
$$

where $\phi(a, b ; z)$ is the Bessel-Maitland function ([15], (1.3); [3], 18.1 (27)).

From (2.1), we have

$$
\begin{aligned}
\sum_{n=0}^{\infty} \frac{Z_{n}^{\alpha}(x ; k) t^{n}}{\Gamma(k n+\alpha+1)} & =\sum_{n=0}^{\infty} \sum_{m=0}^{n} \frac{(-1)^{m} x^{k m} t^{n}}{m !(n-m) ! \Gamma(k m+\alpha+1)} \\
& =\sum_{n=0}^{\infty} \sum_{m=0}^{\infty} \frac{(-1)^{m} x^{k m} t^{n+m}}{m ! n ! \Gamma(k m+\alpha+1)} \\
& =\sum_{n=0}^{\infty} \frac{t^{n}}{n !} \sum_{m=0}^{\infty} \frac{\left(-x^{k} t\right)^{m}}{m ! \Gamma(k m+\alpha+1)} \\
& =e^{t} \phi\left(k, \alpha+1 ;-x^{k} t\right)
\end{aligned}
$$

and (2.2) is established.

Denoting $e^{t} \phi\left(k, \alpha+1 ;-x^{k} t\right)$ by $f(x, t)$ we at once find that $f(x, t)$ satisfies the partial differential equation

$$
x \frac{\partial f}{\partial x}-\alpha t \frac{\partial f}{\partial t}+\alpha t f=0 .
$$

Substituting for $f(x, t)$ from (2.2) and equating the coefficients of $t^{n}$, we obtain the differential recurrence relation

$$
x Z_{n}^{\prime \alpha}(x ; k)=n k Z_{n}^{\alpha}(x ; k)-k \frac{\Gamma(k n+\alpha+1)}{\Gamma(k n+\alpha-k+1)} Z_{n-1}^{\alpha}(x ; k),
$$

also obtained by Konhauser ([8], (6)) by direct calculations.

(ii) Schläfli's Contour integral. It is easy to show that

$$
Z_{n}^{\alpha}(x ; k)=\frac{\Gamma(k n+\alpha+1)}{n ! 2 \pi i} \int_{-\infty}^{(0+)} \frac{\left(t^{k}-x^{k}\right)^{n} e^{t} d t}{t^{k n+\alpha+1}} .
$$

For $\frac{1}{2 \pi i} \int_{-\infty}^{(0+)} \frac{\left(t^{k}-x^{k}\right)^{n} e^{t} d t}{t^{k n+\alpha+1}}=\sum_{j=0}^{n}(-1)^{j}\left(\begin{array}{c}n \\ j\end{array}\right) x^{k j} \frac{1}{2 \pi i} \int_{-\infty}^{(0+)} t^{-(k j+\alpha+1)} e^{t} d t$

$$
=\sum_{j=0}^{n} \frac{(-1)^{j}\left(\begin{array}{c}
n \\
j
\end{array}\right) x^{k j}}{\Gamma(k j+\alpha+1)}
$$

using Hankel's formula ([3], 1.6(2)) 


$$
\frac{1}{\Gamma(z)}=\frac{1}{2 \pi i} \int_{-\infty}^{(0+)} e^{t} t^{-z} d t
$$

finally (2.3) follows from (2.1)

For $k=1,(2.3)$ reduces to the known result ([2], p. 269)

$$
L_{n}^{\alpha}(x)=\frac{\Gamma(n+\alpha+1)}{n ! 2 \pi i} \int_{-\infty}^{(0+)}\left(1-\frac{x}{t}\right)^{n} e^{t} \frac{d t}{t^{\alpha+1}} .
$$

If $\alpha$ is also a positive integer than the integrand in (2.3) is a single-valued analytic function of $t$ with the only singularity $t=0$. Hence we can deform the contour into $|t|=b|x|$ and the substitution $t=x u$ then leads to

$$
x^{\alpha} Z_{n}^{\alpha}(x ; k)=\frac{\Gamma(k n+\alpha+1)}{n ! 2 \pi i} \int_{C}\left(u^{k}-1\right)^{n} e^{x u} u^{-(k n+\alpha+1)} d u
$$

where $C$ denotes the circle $|u|=b$. Indeed $C$ may be replaced by any simple closed contour surrounding the point $u=0$. For $k=2$, (2.5) reduces to the integral representation by Preiser ([11], (5.22)).

Using (2.5), it follows that

$$
\frac{\partial^{k}}{\partial x^{k}}\left[\frac{n ! x^{\alpha+k} Z_{n}^{\alpha+k}(x ; k)}{\Gamma(k n+k+\alpha+1)}\right]=\frac{n ! x^{\alpha} Z_{n}^{\alpha}(x ; k)}{\Gamma(k n+\alpha+1)}
$$

and $\left(\frac{\partial^{k}}{\partial x^{k}}-1\right)\left[\frac{n ! x^{\alpha+k} Z_{n}^{\alpha+k}(x ; k)}{\Gamma(k n+k+\alpha+1)}\right]=\frac{(n+1) ! x^{\alpha} Z_{n+1}^{\alpha}(x ; k)}{\Gamma(k n+k+\alpha+1)}$

which leads to the pure recurrence relation

$$
x^{k} Z_{n}^{\alpha+k}(x ; k)=(k n+\alpha+1)_{k} Z_{n}^{\alpha}(x ; k)-(n+1) Z_{n+1}^{\alpha}(x ; k) .
$$

For $k=2,(2.6)$ reduces to $([11],(5.39))$.

(iii) Fractional integrals and derivatives. We show that

$$
I^{\mu}\left[x^{\alpha} Z_{n}^{\alpha}(x ; k)\right]=\frac{\Gamma(k n+\alpha+1)}{\Gamma(k n+\alpha+\mu+1)} x^{\alpha+\mu} Z_{n}^{\alpha+\mu}(x ; k)
$$

for $\operatorname{Re} \alpha>-1$ and $\operatorname{Re} \mu>-\operatorname{Re}(1+\alpha)$ where for suitable $f$ and complex $\mu, I^{\mu} f(x)$ denotes the $\mu$ th order fractional integral (or fractional derivative) of $f(x)$ (see [10], $\S 2$ ).

When $\operatorname{Re} \mu>0$, we write [10]

$$
\begin{aligned}
I^{\mu}\left[x^{\alpha} Z_{n}^{\alpha}(x ; k)\right] & =\int_{0}^{x} \frac{(x-t)^{\mu-1}}{\Gamma(\mu)} t^{\alpha} Z_{n}^{\alpha}(t ; k) d t \\
& =\frac{\Gamma(k n+\alpha+1)}{n ! \Gamma(\mu)} \sum_{j=0}^{n} \frac{(-n)_{j}}{\Gamma(k j+\alpha+1)} \int_{0}^{x} t^{k j+\alpha}(x-t)^{\mu-1} d t
\end{aligned}
$$




$$
=\frac{\Gamma(k n+\alpha+1)}{n !} \sum_{j=0}^{n}(-n)_{j} \frac{x^{k j+\alpha+\mu}}{\Gamma(k j+\alpha+\mu+1)} \text {; }
$$

hence for $\operatorname{Re} \mu>0$ and $\operatorname{Re} \alpha>-1$, we obtain

$$
I^{\mu}\left[x^{\alpha} Z_{n}^{\alpha}(x ; \mathrm{k})\right]=\frac{\Gamma(k n+\alpha+1)}{\Gamma(k n+\alpha+\mu+1)} x^{\alpha+\mu} Z_{n}^{\alpha+\mu}(x ; k) .
$$

But (2.8) may be written as

$$
x^{\alpha} Z_{n}^{\alpha}(x ; k)=\frac{\Gamma(k n+\alpha+1)}{\Gamma(k n+\alpha+\mu+1)} I^{-\mu}\left[x^{\alpha+\mu} Z_{n}^{\alpha+\mu}(x ; k)\right],
$$

the inversion being valid for $\operatorname{Re} \mu>0$ and the assumptions made.

Putting $\mu^{\prime}=-\mu, \alpha^{\prime}=\alpha+\mu$, we obtain for $\operatorname{Re} \mu^{\prime}<0$

$$
x^{\alpha^{\prime}+\mu^{\prime}} Z_{n}^{\alpha^{\prime}+\mu^{\prime}}(x ; k)=\frac{\Gamma\left(k n+\alpha^{\prime}+\mu^{\prime}+1\right)}{\Gamma\left(k n+\alpha^{\prime}+1\right)} I^{\mu^{\prime}}\left[x^{\alpha^{\prime}} Z_{n}^{\alpha^{\prime}}(x ; k)\right]
$$

which is (4.1) with the letters $\alpha, \mu$ accented. Ignoring the accents we can write

$$
I^{\mu}\left[x^{\alpha} Z_{n}^{\alpha}(x ; k)\right]=\frac{\Gamma(k n+\alpha+1)}{\Gamma(k n+\alpha+\mu+1)} x^{\alpha+\mu} Z_{n}^{\alpha+\mu}(x ; k)
$$

for $\operatorname{Re} \mu<0, \operatorname{Re} \alpha>-1$ and $\operatorname{Re}(\alpha+\mu)>-1$.

When $\operatorname{Re} \mu=0$, we write $I^{\mu}=I^{\mu+1} I^{-1}$ and the result easily follows; thus (2.7) is established for all complex $\mu$ with $\operatorname{Re} \mu>-\operatorname{Re}(1+\alpha)$.

REMARK 1. When $\mu$ is a negative integer say $-m$, then (2.7) is written as

$$
\left(\frac{d}{d x}\right)^{m}\left[x^{\alpha} Z_{n}^{\alpha}(x ; k)\right]=\frac{\Gamma(k n+\alpha+1)}{\Gamma(k n+\alpha-m+1)} x^{\alpha-m} Z_{n}^{\alpha-m}(x ; k)
$$

which can also be proved by direct differentiation provided $\operatorname{Re} \alpha>m-1$.

REMARK 2. For $k=1$, (2.7) unifies the results ([3], 10.12(27)) and ([4], 13.1(49)) for Laguerre polynomials.

3. A singular integral equation. We show that the convolution equation

$$
\int_{0}^{x}(x-t)^{\alpha} Z_{n}^{\alpha}(\lambda(x-t) ; k) f(t) d t=g(x)
$$

for $\operatorname{Re} \alpha>-1$ admits a locally integrable solution $f$ given by 
(3.2) $f(x)=\frac{n !}{\Gamma(k n+\alpha+1)} \int_{0}^{x}(x-t)^{l-\alpha-2} E_{k, l-\alpha-1}^{n}(\lambda(x-t))^{k} I^{-l} g(t) d t$

provided $I^{-l} g$ exists for $\operatorname{Re} l>\operatorname{Re} \alpha+1$ and is locally integrable in $(0, \delta), 0<x<\delta<\infty$.

The function

$$
E_{a, b}^{c}(z)=\sum_{j=0}^{\infty} \frac{(c)_{j} z^{j}}{\Gamma(a j+b) j !} \quad \operatorname{Re} a>0
$$

is a very special case of the generalized hypergeometric functions considered by Wright [16] and is also expressible as a Fox's $H$ function [5]. On the other hand $E_{a, b}^{c}(z)$ is a most natural generalization of the Mittag-Leffler's function $E_{\alpha}(z)([3], 18.1$; [9]) and also contains the confluent hypergeometric function ${ }_{1} F_{1}(c ; d ; z)([3]$, ch.VI), the Wiman's function $E_{a, b}(z)([3], 18.1(19))$ and several other fuctions as special cases. It is an entire function of order $(\operatorname{Re} a)^{-1}$ and indeed has a number of properties which may be of independent interest. A fact of immediate interest to us is that the polynomials $Z_{n}^{\alpha}(x ; k)$ bear to $E_{a, b}^{c}(x)$ a relation which is analogous to that which the Laguerre polynomials $L_{n}^{\alpha}(x)$ bear to the confluent hypergeometric function ${ }_{1} F_{1}$; evidently

$$
Z_{n}^{\alpha}(x ; k)=\frac{\Gamma(k n+\alpha+1)}{n !} E_{k, \alpha+1}^{-n}\left(x^{k}\right) .
$$

As usual let

$$
L[f(t)]=\hat{f}(p)=\int_{0}^{\infty} e^{-p t} f(t) d t \quad \operatorname{Re} p>0
$$

denote the Laplace transform of $f$. Then it is easily verified that for $\operatorname{Re} \lambda, \operatorname{Re} p>0$,

$$
\begin{aligned}
& L\left[t^{b-1} E_{a, b}^{c}(\lambda t)^{a}\right]=p^{-b+a c}\left(p^{a}-\lambda^{a}\right)^{-c} \quad \operatorname{Re} b>0, \\
& L\left[t^{\alpha} Z_{n}^{\alpha}(\lambda t ; k)\right]=\frac{\Gamma(k n+\alpha+1)}{n ! p^{k n+\alpha+1}}\left(p^{k}-\lambda^{k}\right)^{n}, \quad \operatorname{Re} \alpha>-1 .
\end{aligned}
$$

We next note a general result on the Laplace transform of the $r$-times repeated indefinite integral as well as the $r$ th order derivative of a function; in fact, we observe that

$$
p^{\mu} \hat{f}(p)=L\left[I^{-\mu} f(t)\right]
$$

for suitable $f$, complex $\mu$ and $p$ with $\operatorname{Re} p>0$. Evidently both ([4], 4.1(8)) and ([4], 4.1(9)) are included in (3.8) as special cases.

We are now prepared to solve (3.1). From (3.1), (3.4) and using $([4], 4.1(20))$, we have 


$$
\frac{\Gamma(k n+\alpha+1)}{n !}\left(p^{k}-\lambda^{k}\right)^{n} p^{-k n-\alpha-1} \hat{f}(p)=\hat{g}(p) .
$$

For $\operatorname{Re} l>\operatorname{Re}(\alpha+1)$, (3.9) can be written (compare with [1]) as

$$
\hat{f}(p)=\frac{n !}{\Gamma(k n+\alpha+1)}\left\{\left(p^{k}-\lambda^{k}\right)^{-n} p^{-l+k n+\alpha+1}\right\}\left\{p^{l} \hat{g}(p)\right\}
$$

and we finally get

$$
f(x)=\frac{n !}{\Gamma(k n+\alpha+1)} \int_{0}^{x}(x-t)^{l-\alpha-2} E_{k, l-\alpha-1}^{n}(\lambda(x-t))^{k} I^{-l} g(t) d t
$$

using ([4], 4.1(20)), (3.6) and (3.8).

4. A general class of generating functions. For arbitrary $\lambda$, we prove the generating relation

$$
(1-t)^{-\lambda} E_{k, \alpha+1}^{\lambda}\left(\frac{-x^{k} t}{1-t}\right)=\sum_{n=0}^{\infty} \frac{(\lambda)_{n} Z_{n}^{\alpha}(x ; k) t^{n}}{\Gamma(k n+\alpha+1)} .
$$

From (2.1), we have

$$
\begin{aligned}
\sum_{n=0}^{\infty} \frac{(\lambda)_{n} Z_{n}^{\alpha}(x ; k) t^{n}}{\Gamma(k n+\alpha+1)} & =\sum_{n=0}^{\infty} \sum_{m=0}^{n} \frac{(-1)^{m}(\lambda)_{n} x^{k m} t^{n}}{\Gamma(k m+\alpha+1)(n-m) ! m_{n}^{!} !} \\
& =\sum_{n=0}^{\infty} \sum_{m=0}^{\infty} \frac{(-1)^{m}(\lambda)_{n+m} x^{k m} t^{n+m}}{\Gamma(k m+\alpha+1) n ! m !} \\
& =\sum_{m=0}^{\infty} \frac{(\lambda)_{m}\left(-x^{k} t\right)^{m}}{\Gamma(k m+\alpha+1) m !} \sum_{n=0}^{\infty} \frac{(\lambda+m)_{n} t^{n}}{n !} \\
& =(1-t)^{-\lambda} E_{k, \alpha+1}^{\lambda}\left(\frac{-x^{k} t}{1-t}\right) .
\end{aligned}
$$

For $k=1, \lambda=1+\alpha$, (4.1) yields the well-known generating function ([3], 10.12(7)) for the Laguerre polynomials.

From (4.1) we obtain, on applying Taylor's theorem

$$
\frac{(\lambda)_{n} Z_{n}^{\alpha}(x ; k)}{\Gamma(k n+\alpha+1)}=\frac{1}{2 \pi i} \int_{C}(1-t)^{-\lambda} E_{k, \alpha+1}^{\lambda}\left(\frac{-x^{k} t}{1-t}\right) t^{-n-1} d t,
$$

$C$ being a closed contour surrounding $t=0$ and lying within the disk $|t|<1$. Putting $u=x^{k} / 1-t$,

$$
x^{k \lambda-k} Z_{n}^{\alpha}(x ; k)=\frac{\Gamma(k n+\alpha+1)}{(\lambda)_{n} 2 \pi i} \int_{C^{\prime}} \frac{u^{n+\lambda-1} E_{\hat{k}, \alpha+1}^{\lambda}\left(x^{k}-u\right) d u}{\left(u-x^{k}\right)^{n+1}}
$$

where $C^{\prime}$ is a circle $\left|u-x^{k}\right|=\rho$ of small radius $\rho$.

Choosing $\lambda=1$, we have in terms of Wiman's function 


$$
Z_{n}^{\alpha}(x ; k)=\frac{\Gamma(k n+\alpha+1)}{n ! 2 \pi i} \int_{C^{\prime}} \frac{u^{n} E_{k, \alpha+1}\left(x^{k}-u\right) d u}{\left(u-x^{k}\right)^{n+1}} .
$$

Also evaluating the integral (4.3) by the Cauchy's residue theorem, we obtain for arbitrary $\lambda$ with $\operatorname{Re} \lambda>0$,

$$
Z_{n}^{\alpha}(x ; k)=\frac{\Gamma(k n+\alpha+1)}{(\lambda)_{n} n !} x^{k-k \lambda} \frac{\partial^{n}}{\partial u^{n}}\left[u^{\lambda+n-1} E_{k, \alpha+1}^{\lambda}\left(x^{k}-u\right)\right]_{u=x^{k}}
$$

Since $E_{1, b}^{b}(z)=(1 / \Gamma(b)) e^{z}$, for $\mathrm{k}=1$ and $\lambda=\alpha+1$, (4.5) reduces to the Rodrigues for the Laguerre polynomials.

I am grateful to Professor U. N. Singh for his encoragement and interest in this work.

\section{REFERENCES}

1. R. G. Buschman, Convolution equations with generalized Laguerre polynomial kernels, SIAM Review 6 (1962), 166-167.

2. E. T. Copson, Theory of functions of a complex variable, Oxford University Press, 1961.

3. A. Erdélyi et al., Higher transcendental functions, vols. 1-3, McGraw-Hill, New York, 1953.

4. - Tables of integral transforms, vols. 1-2, McGraw-Hill, New York, 1954.

5. Charles Fox, $G$ and $H$ functions as symmetrical Fourier kernels, Trans. Amer. Math. Soc. 98 (1961), 395-429.

6. P. R. Khandekar, On a convolution transform involving generalized Laguerre polynomial as its kernel, J. Math. Pure Appl. (9) 44 (1965), 195-197.

7. Joseph D. E. Konhauser, Some properties of biorthogonal polynomials, J. Math. Anal. Appl. 11 (1965), 242-260.

8. ㄴ. Biorthogonal polynomials suggested by the Laguerre polynomials, Pacific J. Math. 21 (1967), 303-314.

9. G. M. Mittag-Leffler, Sur la représentation analytique d'une branche uniforme d'une fonction monogène, Acta Math. 29 (1905), 101-182.

10. Tilak Raj Prabhakar, Two singular integral equations involving confluent hypergeometric functions, Proc. Cambridge Phil. Soc. 66 (1969), 71-89.

11. S. Preiser, An investigation of biorthogonal polynomials derivable from ordinary differential equations of the third order, J. Math. Anal. Appl. 4 (1962), 38-64.

12. K. C. Rusia, An integral equation involving generalized Laguerre polynomial, Math. Japon. 11 (1966), 15-18.

13. L. Spencer and Fano, Penetration and diffusion of X-rays, calculation of spatial distributions by polynomial expansion, J. Research, National Bureau of Standards 46 (1951), 446-461.

14. D. V. Widder, The inversion of a convolution transform whose kernel is a Laguerre polynomial, Amer. Math. Monthly 70 (1963), 291-293.

15. E. M. Wright, On the coefficients of power series having exponential singularities, J. London Math. Soc. 8 (1933), 71-79.

16. The asymptotic expansion of the generalized hypergeometric function, Proc. London Math. Soc. 46 (1940), 389-408.

Received January 13, 1970.

\section{RAMJAS COLLEge}

UNIVERSITY OF DELHI

DELHI-7. (INDIA) 



\section{PACIFIC JOURNAL OF MATHEMATICS}

\section{EDITORS}

H. SAmelson

Stanford University

Stanford, California 94305

RICHARd PieRCe

University of Washington

Seattle, Washington 98105

\author{
J. DugundJI \\ Department of Mathematics \\ University of Southern California \\ Los Angeles, California 90007 \\ RICHARD ARENS \\ University of California \\ Los Angeles, California 90024
}

ASSOCIATE EDITORS
E. F. BECKENBACH
B. H. NeumanN
F. WOLF
K. YosHIDA

\section{SUPPORTING INSTITUTIONS}

UNIVERSITY OF BRITISH COLUMBIA

CALIFORNIA INSTITUTE OF TECHNOLOGY

UNIVERSITY OF CALIFORNIA

MONTANA STATE UNIVERSITY

UNIVERSITY OF NEVADA

NEW MEXICO STATE UNIVERSITY

OREGON STATE UNIVERSITY

UNIVERSITY OF OREGON

OSAKA UNIVERSITY

UNIVERSITY OF SOUTHERN CALIFORNIA
STANFORD UNIVERSITY

UNIVERSITY OF TOKYO

UNIVERSITY OF UTAH

WASHINGTON STATE UNIVERSITY

UNIVERSITY OF WASHINGTON

AMERICAN MATHEMATICAL SOCIETY CHEVRON RESEARCH CORPORATION TRW SYSTEMS

NAVAL WEAPONS CENTER 


\section{Pacific Journal of Mathematics}

\section{Vol. 35, No. $1 \quad$ September, 1970}

B. D. Arendt and C. J. Stuth, On the structure of commutative periodic semigroups ..................................... 1

B. D. Arendt and C. J. Stuth, On partial homomorphisms of semigroups .... 7

Leonard Asimow, Extensions of continuous affine functions ............ 11

Claude Elias Billigheimer, Regular boundary problems for a five-term recurrence relation ................................... 23

Edwin Ogilvie Buchman and F. A. Valentine, A characterization of the

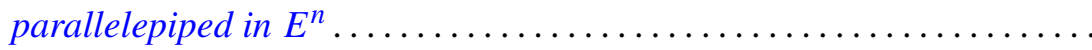
53

Victor P. Camillo, A note on commutative injective rings ............ 59

Larry Jean Cummings, Decomposable symmetric tensors ............. 65

J. E. H. Elliott, On matrices with a restricted number of diagonal values ... 79

Garth Ian Gaudry, Bad behavior and inclusion results for multipliers of type

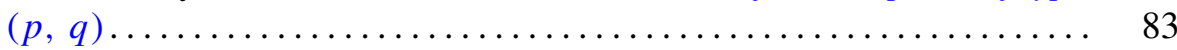

Frances F. Gulick, Derivations and actions .................. 95

Langdon Frank Harris, On subgroups of prime power index . . . . . . . . 117

Jutta Hausen, The hypo residuum of the automorphism group of an abelian

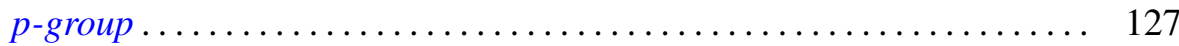

R. Hrycay, Noncontinuous multifuctions . ................... 141

A. Jeanne LaDuke, On a certain generalization of $p$ spaces ............ 155

Marion-Josephine Lim, Rank preservers of skew-symmetric matrices...... 169

John Hathway Lindsey, II, On a six dimensional projective representation of the Hall-Janko group ................................... 175

Roger McCann, Transversally perturbed planar dynamical systems . . . . . 187

Theodore Windle Palmer, Real $C^{*}$-algebras ................... 195

Don David Porter, Symplectic bordism, Stiefel-Whitney numbers, and a

Novikov resolution ....................................... 205

Tilak Raj Prabhakar, On a set of polynomials suggested by Laguerre

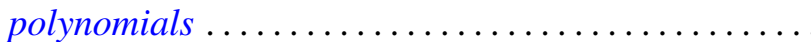

B. L. S. Prakasa Rao, Infinitely divisible characteristic functionals on locally convex topological vector spaces...................

John Robert Reay, Caratheodory theorems in convex product structures ...

Allan M. Sinclair, Eigenvalues in the boundary of the numerical range. .

David R. Stone, Torsion-free and divisible modules over matrix rings ....

William Jennings Wickless, A characterization of the nil radical of a

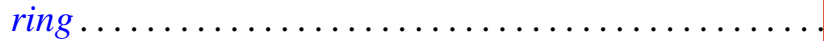

